\title{
Liquid methanol sorption, diffusion and permeation in charged and uncharged polymers
}

\author{
Michele Galizia, Donald R. Paul and Benny D. Freeman* \\ J. McKetta Department of Chemical Engineering, 200 E. Dean Keeton Street, 78712 Austin, \\ and Center for Energy and Environmental Resources, 10100 Burnet Road, 78758 Austin, \\ The University of Texas at Austin, Texas (USA)
}

\section{Revised manuscript}

*Corresponding author: B.D. Freeman, freeman@che.utexas.edu ph. (512)232 2803 


\section{Graphical abstract (TOC)}

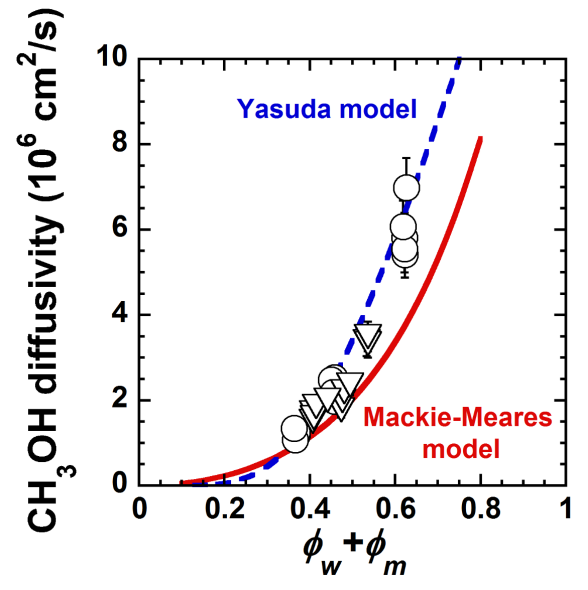




\begin{abstract}
There are few studies of the impact of membrane charge on ion and organic uncharged solute transport in polymers. This study was aimed at addressing the lack of such fundamental information in the literature. Liquid methanol sorption, diffusion and permeation in water swollen, cross-linked, uncharged poly(ethylene glycol) diacrylate (XLPEGDA) and in charged membranes were investigated. Membrane water content significantly influences methanol solubility, diffusivity and permeability, with more swollen membranes having faster methanol transport rates. The Mackie-Meares model was used to interpret and correlate diffusion data. The Flory-Rehner theory for ternary systems was used to interpret methanol sorption isotherms in XLPEGDA-based polymers. Methanol and sodium chloride transport patterns are similar in neutral hydrogels, while substantial differences are observed in ion exchange polymers. These differences are ascribed to sodium chloride transport in charged polymers being strongly influenced by Donnan exclusion, while methanol sorption and diffusion are not.
\end{abstract}

Keywords: diffusion; swollen polymers; Mackie-Meares model. 


\section{Introduction}

Fundamental understanding of small molecules transport in hydrated or solvent swollen polymers plays a key role in the design of new polymers for many applications, such as membrane separations, controlled drug delivery and tissue engineering $[1,2,3,4,5]$. Ion exchange membranes (IEMs) are typically cross-linked and have ionizable groups attached to their backbones [6]. Even though these materials often have a hydrophobic hydrocarbon structure, the presence of fixed charges, such as sulfonate or quaternary ammonium groups, gives them a hydrophilic character, and, as a result, such materials often sorb relatively large amounts of water, which has a substantial impact on transport properties of both neutral and charged solutes [6,7,8]. Ion exchange membranes are used in many applications, such as water purification via electrically driven processes, like electrodialysis [9]. Electrodialysis exploits the selective permeability of IEMs to cations or anions to separate ions from water. High selectivity for ions of different charge stems from Donnan exclusion of ions having the same charge as that of the fixed ions on the IEM backbone [6,7].

Due to their greater ionic conductivity and lower methanol permeability relative to perfluoro-sulfonated ionomers, hydrocarbon-based IEMs have been explored as potential electrolytes for direct methanol fuel cells (DMFCs) [10,11,12,13]. Additionally, recently proposed applications, such as artificial photosynthesis, require ion exchange materials that are both highly conductive to ions and have good barrier properties to uncharged molecules, such as methanol $[14,15]$. These applications require fundamental understanding of small molecule transport in swollen polymers. 
Cross-linked poly(ethylene glycol) diacrylate (XLPEGDA) is a neutral hydrogel selected to serve as a model uncharged material to compare with the IEMs considered in this study. Its water uptake often exceeds 50 wt. \% and can be tuned by, for example, adding water to the prepolymerization mixture [16]. Thus, it represents a convenient uncharged, cross-linked material having water content values in the same range as those of some commercial IEMs.

Neutral molecules sorb into uncharged polymers until the penetrant chemical potential in the external phase equals that in the polymer/penetrant mixture $[8,17,18]$. Similarly, when an uncharged polymer is soaked in a water/salt solution, such thermodynamic partitioning governs ion sorption and, for monovalent salts, an equivalent molar amount of anions and cations sorb into the polymer to preserve electro-neutrality $[8,19]$.

Conversely, in charged polymers, ion sorption may be strongly influenced by the presence of fixed charges on the polymer backbone $[8,20]$. In a cation exchange polymer, for example, negatively charged groups (e.g., sulfonate groups) are attached to the polymer backbone, and the concentration of sorbed cations (counter-ions) can significantly exceed the concentration of sorbed anions (co-ions) [8]. The majority of sorbed cations are associated with the fixed charges, and additional sorbed cations are present to neutralize mobile anions sorbed into the membrane [9]. Due to the unequal distribution of ions between the swollen polymer and the contiguous external solution, an electric potential difference (the so-called Donnan potential) exists at the interface between the polymer and the solution [6]. This potential difference inhibits co-ion sorption in the membrane and counter-ion desorption into the contiguous external solution. This effect is known as Donnan exclusion. Sorption equilibrium is 
attained when the electrochemical potential of each type of ion (i.e., cations and anions) in the external solution is equal to the corresponding electrochemical potentials of those species in the polymer-penetrant mixture [6]. The importance of Donnan exclusion on salt transport in charged polymers can be observed by a strong increase in salt sorption and permeability coefficients with increasing external salt concentration, $C_{s}^{s}$ [8]. As external salt concentration increases, Donnan exclusion is gradually overwhelmed, so the material exhibits sorption properties more like those of an uncharged polymer $[8,19,20]$.

In this study, methanol was selected as a model uncharged probe molecule for sorption and permeation measurements in part because of its complete solubility in water over the whole range of compositions, and in part because of its greater diffusion rate relative to other organic liquids. The extent to which methanol transport in membranes is reduced relative to that in pure water is discussed and related to membrane water content. The diffusion coefficient of liquid methanol in water-swollen hydrogels is compared to that in pure water and in glassy thermoplastics, such as poly(ethylene terephthalate) (PET) and polystyrene (PS). Finally, methanol transport in charged and uncharged hydrated polymers was compared with sodium chloride transport in the same materials.

\section{Theoretical background}

2.1. Solute transport in swollen polymers. Permeability, $P_{i}$, of a liquid penetrant, $i$, through a swollen polymer film is defined as follows [21]:

$$
P_{i}=\frac{N_{i} \ell}{\Delta C_{i}}
$$


where $N_{i}$ is the steady-state penetrant flux through the film, $\ell$ is the swollen film thickness, and $\Delta C_{i}$ is the penetrant concentration difference in the external solutions on either side of the film. Permeability coefficients calculated according to Eq. 1 are expressed in units $\mathrm{of}_{\mathrm{cm}}^{2} / \mathrm{s}$. The solubility (or partition) coefficient, $K_{i}$, is the ratio of the equilibrium concentration of solute inside the membrane $\left(C_{i}^{m}\right)$ to that in the contiguous external solution $\left(C_{i}^{s}\right)[8]$ :

$$
K_{i}=\frac{C_{i}^{m}}{C_{i}^{s}}
$$

The concentrations appearing in Eq. 2 are expressed in (mol of penetrant)/(volume of swollen polymer).

Based on the solution-diffusion model, diffusivity, solubility and permeability are related as follows [22]:

$$
P_{i}=\bar{D}_{i} \times K_{i}
$$

where $\bar{D}_{i}$ is the average, effective diffusion coefficient of the penetrant in the polymer. From Eq. 2, the solubility coefficient is dimensionless, so diffusivity has the same units of permeability (i.e., $\left.\mathrm{cm}^{2} / \mathrm{s}\right)$.

2.2. Mackie-Meares Model. In highly swollen polymer films, absent specific polymer-penetrant interactions, the primary means by which the polymer chains influence penetrant transport is by acting as impenetrable obstacles, thereby reducing the effective area available for transport and increasing the path length required by a penetrant molecule to cross a film of a given thickness (i.e., increasing tortuosity) [23]. Due to these tortuosity/area effects, small molecule 
diffusion through such swollen membranes is reduced relative to that in pure solvent. The Mackie-Meares model relates the diffusion coefficient of a penetrant through solvent, $D_{0, i}$, to the diffusion coefficient in a solvent-swollen membrane, $\bar{D}_{i}$, by the following expression, which depends only on the polymer volume fraction, $\phi_{p}[23]$ :

$\bar{D}_{i}=D_{0, i}\left(\frac{1-\phi_{p}}{1+\phi_{p}}\right)^{2}$

$D_{0, i}$ can be obtained from literature sources or estimated using the Wilke-Chang correlation [24]:

$$
D_{0, i}=7.4 \cdot 10^{-8} \frac{T\left(\psi_{w} M_{w}\right)^{0.5}}{\eta_{w} \tilde{V}_{m}^{0.6}}
$$

where $T$ is temperature $\left(298.15 \mathrm{~K}\right.$ in this study), $M_{w}$ is the solvent (i.e., water in our case) molecular weight $(18 \mathrm{~g} / \mathrm{mol}), \eta_{w}$ is the solvent viscosity $(0.8937 \mathrm{cP})[25], \tilde{V}_{m}$ is the solute molar volume $\left(40.46 \mathrm{~cm}^{3} / \mathrm{mol}\right.$ for methanol) [25] and $\Psi_{w}$ is the solvent association parameter (2.6 for water, 1 for unassociated liquids) [24]. The methanol diffusion coefficient in water, calculated using this approach, is $1.7 \times 10^{-5} \mathrm{~cm}^{2} / \mathrm{s}$, which is in good agreement with the experimental value of $1.6 \times 10^{-5} \mathrm{~cm}^{2} / \mathrm{s}$ reported by Lee et al. [26].

2.3. Yasuda's Model. Free volume theory is commonly used to describe penetrant diffusion in polymers [27]. Yasuda et al. [28,29] proposed that the free volume available for solute transport in water-swollen polymers is proportional to the water content. In this view, solute diffusivity 
depends exponentially on reciprocal free volume, $v_{f}$, according to the following expression [28]:

$\bar{D}_{i} \propto \exp \left(-\frac{v^{*}}{v_{f}}\right)$

where $v^{*}$ is the minimum size of a free volume hole required to enable penetrants to perform a diffusion jump, and $v_{f}$ is the free volume available for solute transport. If $v_{f}$ is proportional to the volume fraction of water in the polymer, $\phi_{w}$, as Yasuda et al. [28] suggested, then:

$v_{f}=v_{f, w} \phi_{w}$

where $v_{f, w}$ is the free volume in pure water. As a result, Eq. 6 can be written as follows:

$\bar{D}_{i}=D_{0, i} \exp \left[-A\left(\frac{1}{\phi_{w}}-1\right)\right]$

where $D_{0, i}$ is the solute diffusivity in pure solvent, and $A$ is an empirical model constant. Eq. 8 predicts a linear relationship between the logarithm of solute diffusivity and the reciprocal of membrane water content. As $\phi_{w}$ approaches unity, solute diffusivity $\bar{D}_{i}$ approaches its value in pure water, $D_{0, i}$. Like the Mackie-Meares model, the Yasuda model predicts that solute diffusion coefficients go to zero as polymer volume fraction goes to 1, so both models break down at high polymer volume fractions.

2.4. Flory-Rehner model. The Flory-Rehner model has been widely used to describe and correlate gas, vapor and liquid sorption in rubbery cross-linked polymers [30,31]. The approach 
proposed by Flory and Rehner modifies the classical Flory-Huggins equation by introducing an elastic term to account for the presence of cross-links [30,31]. To describe methanol sorption in a water swollen film, the Flory-Rehner model for ternary systems [30,31,32] is used, since the mixture comprises three components: polymer, water and methanol. To calculate methanol sorption isotherms in swollen XLPEGDA films, the following system of three equations must be solved [30,31]:

$$
\left\{\begin{array}{l}
\ln a_{m}=\ln \phi_{m}+\left(1-\phi_{m}\right)-\frac{\tilde{V}_{m}}{\tilde{V}_{w}} \phi_{w}-\frac{\tilde{V}_{m}}{\tilde{V}_{p}} \phi_{p}+\left(\chi_{m w} \phi_{w}+\chi_{m p} \phi_{p}\right)\left(\phi_{w}+\phi_{p}\right)-\chi_{w p} \frac{\tilde{V}_{m}}{\tilde{V}_{w}} \phi_{w} \phi_{p}+\tilde{V}_{m} \nu_{c}\left(\phi_{p}^{1 / 3}-\frac{\phi_{p}}{2}\right) \\
\ln a_{w}=\ln \phi_{w}+\left(1-\phi_{w}\right)-\frac{\tilde{V}_{w}}{\tilde{V}_{m}} \phi_{m}-\frac{\tilde{V}_{w}}{\tilde{V}_{p}} \phi_{p}+\left(\chi_{m w} \phi_{m} \frac{\tilde{V}_{w}}{\tilde{V}_{m}}+\chi_{w p} \phi_{p}\right)\left(\phi_{m}+\phi_{p}\right)-\chi_{m p} \frac{\tilde{V}_{w}}{\tilde{V}_{m}} \phi_{m} \phi_{p}+\tilde{V}_{w} \nu_{c}\left(\phi_{p}^{1 / 3}-\frac{\phi_{p}}{2}\right) \\
\phi_{m}+\phi_{w}+\phi_{p}=1
\end{array}\right.
$$

where subscripts $m, w$ and $p$ refer to methanol, water and polymer respectively. $a_{m}$ and $a_{w}$ are the activites of methanol and water, respectively, in the external solution, $\phi_{i}$ is the equilibrium volume fraction of component $i$ in the mixture, $v_{c}$ is the cross-link density expressed in $\operatorname{mol}\left(\right.$ cross-link) $/ \mathrm{cm}^{3}$ (polymer), and $\tilde{V}_{i}$ is the molar volume of component $i$. Finally, $\chi_{i, j}$ is the Flory-Huggins parameter, which accounts for binary interaction between species $i$ and $j$. In view of the large size of the polymer relative to the penetrants, in Eq. $9 \frac{\tilde{V}_{m}}{\tilde{V}_{p}} \rightarrow 0$ and $\frac{\tilde{V}_{w}}{\tilde{V}_{p}} \rightarrow 0$ $[30,31,32]$.

Methanol-polymer $\left(\chi_{m p}\right)$ and water-polymer $\left(\chi_{w p}\right)$ binary interaction parameters can be calculated from pure liquid sorption data in the polymer, interpreted using the binary version 
of Flory-Rehner theory [32]. The methanol-water binary parameter, $\chi_{m w}$, has been treated as an adjustable parameter. Finally, the XLPEGDA cross-link density, $v_{c}$, has been taken from literature sources [33]. Values of these parameters are recorded in Table 1. Once the parameters are known, Eqs. 9 represent a non-linear system of three algebraic equations, where $\phi_{m}, \phi_{w}$ and $\phi_{p}$ are the unknowns. This system of equations was solved numerically using Matlab ${ }^{\circledR}$.

Table 1. Methanol-XLPEGDA and water-XLPEGDA Flory-Huggins interaction parameters. Cross-link density, $v_{c}$, was taken from literature sources [33].

\begin{tabular}{|c|c|c|c|}
\hline Sample & $\chi_{m p}$ & $\chi_{w p}$ & $v_{c}\left(\mathrm{~mol} / \mathrm{cm}^{3}\right)$ [33] \\
\hline XLPEGDA_0 & 1.05 & 0.86 & 0.0024 \\
\hline XLPEGDA_20 & 0.87 & 0.75 & 0.0019 \\
\hline XLPEGDA_60 & 0.64 & 0.58 & 0.0014 \\
\hline
\end{tabular}

Note: The Flory-Huggins interaction parameters were calculated from pure liquid sorption data and cross-link density for different samples of cross-linked poly(ethylene glycol diacrylate) (XLPEGDA). The numbers appearing after the XLPEGDA designation refer to the weight percent of deionized water in the pre-polymerization mixture.

\section{Experimental}

3.1. Materials. Dense XLPEGDA films were prepared via UV radical photopolymerization, according to a protocol previously reported [16]. Poly(ethylene glycol diacrylate) (SigmaAldrich, Milwaukee, WI) with an average molecular weight of 700, was mixed with different amounts of deionized water (0, 20 and 60\% wt). 1-hydroxycyclohexyl phenyl ketone (HCPK, Sigma-Aldrich) was used as the photoinitiator ( $0.1 \%$ wt of the pre-polymerization solution). The pre-polymerization solution was spread between two clean quartz plates and cross-linked 
under UV irradiation (wavelength $312 \mathrm{~nm}$ ) for $90 \mathrm{~s}$ at $3 \mathrm{~mW} / \mathrm{cm}^{2}$ in a UV cross-linker (UVXL1000, Spectronic Corporation, Westbury, NY). Metal spacers of defined thickness ( $0.5 \mathrm{~mm})$ were placed between the glass plates to control the thickness of the nascent films. Following polymerization, the films were peeled off the quartz plates and soaked in de-ionized water for $48 \mathrm{~h}$ to remove any impurities, such as unreacted monomer and any other species not incorporated into the resulting cross-linked network. XLPEGDAs films are named XLPEGDA_X, where $X$ represents the weight percent of water in the pre-polymerization solution (i.e., 0, 20 and 60\% wt). As shown in Table 2, these values of pre-polymerization water content yield cross-linked polymers whose water uptake spans the range of water uptake values in the ion exchange polymers considered in this study. The cross-link density of XLPEGDA-based materials was measured by Lin et al. [33] from water swelling experiments, i.e., at conditions that closely mimic those experienced by XLPEGDA samples in this study. Thus, the cross-link density reported in ref. [33] can be safely used to describe liquid methanol sorption in water swollen XLPEGDA.

Commercial ion exchange membranes were kindly provided by General Electric Power and Water (Westborough, MA). CR61 and AR103 membranes are based on a crosslinked poly(styrene-divinylbenzene) structure. CR61 has sulfonate groups attached to the aromatic backbone, so it is a cation exchange membrane. Conversely, AR103 contains quaternary ammonium groups, so it is an anion exchange membrane. AR204 has an acrylic-based backbone with quaternary ammonium groups as fixed charges, so it is also an anion exchange membrane. Nominal values of the ion-exchange capacity (IEC), provided by the manufacturer, are recorded 
in Table 2. The ion exchange membranes used in this work contain a fabric backing, so sorption and transport data have been normalized based on the polymer phase alone, according to a method reported separately [20]. Water and methanol uptake by the backing is negligible relative to the polymer phase. The structures of the polymers are reported in the Supplementary Information (Fig. S1).

Methanol was purchased from Sigma-Aldrich (purity $>99.8 \%$ ) and was used as received. Deionized water (with a specific conductivity of $0.055 \mu \mathrm{S} / \mathrm{cm}$ and a TOC content of about 5.4 ppb) was generated by a Millipore system (RiOS 8, Billerica, MA).

Table 2. Ion exchange capacity and pure liquid water uptake at room conditions.

\begin{tabular}{|c|c|c|c|}
\hline & & $\begin{array}{c}I E C^{\|} \\
(\text {meq/g(dry polymer }))\end{array}$ & $\begin{array}{c}\text { pure water uptake§ } \\
\text { (g water/g(dry polymer) }\end{array}$ \\
\hline \multirow{3}{*}{$\begin{array}{l}\vec{a} \\
\frac{a}{3} \\
z\end{array}$} & XLPEGDA_0 & 0 & $0.49 \pm 0.001$ \\
\hline & XLPEGDA_20 & 0 & $0.71 \pm 0.002$ \\
\hline & XLPEGDA_60 & 0 & $1.39 \pm 0.008$ \\
\hline \multirow{3}{*}{ 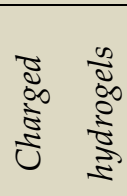 } & AR103 & 2.2 (minimum) & $0.64 \pm 0.003$ \\
\hline & CR61 & 2.2 (minimum) & $0.82 \pm 0.002$ \\
\hline & AR204 & 2.4 (minimum) & $0.99 \pm 0.007$ \\
\hline
\end{tabular}

"This value was reported by General Electric Power and Water.

sThese values were measured in this study.

3.2. Methanol permeability measurements. Methanol permeability through water swollen charged and uncharged membranes was measured at $25^{\circ} \mathrm{C}$ using side-by-side diaphragm permeation cells (PermeGear Inc., Hellertown, PA). Before a permeation test, the membranes were soaked in de-ionized water for $48 \mathrm{~h}$ to ensure attainment of swelling equilibrium. The swollen 
membrane was then sealed between the donor and the receiver compartments: the former was filled with $35 \mathrm{~mL}$ of methanol-water solution, whose methanol content was in the range 0.1-12 $\mathrm{mol} / \mathrm{L}$, while the latter was filled with $35 \mathrm{~mL}$ of pure de-ionized water. Both compartments were water-jacketed to ensure constant temperature during the experiment to within $\pm 0.1^{\circ} \mathrm{C}$. Mixing in both compartments was achieved using magnetic stirrers. Pseudo-steady state methanol flux through the membrane was obtained by tracking methanol concentration in the receiver compartment over time with a Total Organic Carbon (TOC) analyzer (TOC-Vcsh, Shimadzu):

$$
N_{i}=\frac{\dot{Q}}{A} \quad \text { (Eq. 10) }
$$

where $A$ is the membrane area $\left(A \sim 1.77 \mathrm{~cm}^{2}\right)$ and $\dot{Q}$ represents the moles of penetrant transferred across the membrane per unit time at steady-state. The moles of methanol in the receiver compartment were calculated at any time based on TOC analysis and by knowing the total volume of liquid in the compartment. Finally, methanol permeability at each upstream concentration was calculated according to Eq. 1, based on an average of at least four measurements. The results were reproducible with an uncertainty of less than $5 \%$. The film thickness was measured with a Mitutoyo absolute micrometer (Mitutoyo, Aurora, IL). Since XLPEGDA films are very soft, they were sandwiched between two metal plates, whose thickness was known, and the sandwich thickness was measured. The actual membranes thickness was obtained by subtracting the thickness of the metal plates from the thickness of the sandwich structure. 
During the experiments, osmotic effects (i.e., water transfer from the receiver to donor compartment) can take place when the methanol concentration gradient across the membrane is significant [34]. The methanol concentration in the donor compartment $\left(C_{m}^{s}\right)$ was measured via TOC analysis at the end of each experiment and was used to calculate methanol permeability through Eq. 1. Indeed, $C_{m}^{s}$ changes over time due to the osmotic water transfer into the donor compartment and to the methanol diffusion into the receiver compartment. However, the change in $C_{m}^{s}$ between the beginning and the end of an experiment is less than $4 \%$. The issue of osmotic water transfer is addressed in the Supplementary Information.

3.3. Sorption measurements. Membranes for sorption experiments were cut from the original film as small disks, whose diameter ranged from 2.5 to $3.5 \mathrm{~cm}$. Before sorption tests, they were swollen in pure de-ionized water for $48 \mathrm{~h}$ at ambient conditions.

Swollen membranes were removed from de-ionized water, quickly blotted with paper towels and soaked for 3 days in methanol-water solutions, to ensure attainment of sorption equilibrium. The soaking solution was changed several times during the experiment. The membranes were then removed from the soaking solution, quickly blotted with a paper tissue and weighed with an analytical balance (Mettler Toledo, accuracy $10^{-4} \mathrm{~g}$ ). After recording their weight, $m_{w}$, the membranes were soaked again in $25 \mathrm{~mL}$ of pure deionized water for 3 days, and methanol was allowed to desorb. The amount of methanol sorbed by each membrane was estimated by analyzing the composition of the desorption solution via TOC analysis. To ensure that sorbed methanol had been completely removed, the membranes were soaked again in DI water for 2 days, and the desorption solution composition was measured. No trace of methanol 
was found at the end of the second desorption run. Methanol sorption isotherms were obtained by normalizing the moles of sorbed methanol by the volume of swollen polymer. The latter was calculated based on the thickness and diameter of the wet membrane. The membrane diameter was measured using an electronic caliper (Fowler Instruments, Boston, MA). The volume fraction of methanol was calculated using the following equation:

$\phi_{m}=\frac{C_{m} \bar{V}_{m}}{\sum_{i} C_{i} \bar{V}_{i}}$

where $C_{i}$ represents the concentration of species $i$, expressed as moles of species $i$ per unit volume of dry polymer at the operating temperature, and $\bar{V}_{i}$ represents the partial molar volume of species $i$. Each sorption experiment was repeated multiple times, with a reproducibility of about $4 \%$. Sorption experiments were also repeated using longer equilibration times in water-methanol solutions, obtaining the same results. Thus, an equilibration time of 3 days seems to be adequate.

After methanol desorption, the membranes were dried at room temperature in a vacuum oven until reaching a constant dry mass $m_{D}$. Finally, the total liquid uptake, $W$, was calculated as follows:

$W=\frac{m_{W}-m_{D}}{m_{D}}$

Water and methanol uptake were calculated from the mass balance. The same protocol was used to estimate pure water and pure methanol uptake at $25^{\circ} \mathrm{C}$. 


\section{Results and Discussion}

4.1. Total mass uptake. In Fig. 1-A, water/methanol mixture sorption isotherms at $25^{\circ} \mathrm{C}$ are reported for uncharged XLPEGDAs as a function of external methanol concentration. The external methanol concentration ranged from $0 \mathrm{~mol} / \mathrm{L}$ (pure water) to $24.7 \mathrm{~mol} / \mathrm{L}$ (pure methanol). For all samples, uptake decreases with increasing methanol concentration in the external solution, indicating that these samples have a greater thermodynamic affinity for water than for methanol. The percent decrease in total uptake over the entire range of external methanol concentration is about 130\% for XLPEGDA_0, 110\% for XLPEGDA_20 and 105\% for XLPEGDA_60.

At any given external solution concentration, the total uptake increases with increasing amount of water in the pre-polymerization solution (i.e., XLPEGDA_60 > XLPEGDA_20 > XLPEGDA_0). As the amount of water in the pre-polymerization solution increases, the crosslink density decreases (cf., Table 1), which permits more penetrant sorption. Similar results were reported by Lin et al. [33] when considering gas sorption and permeation in XLPEGDAbased materials having different pre-polymerization water content values. The pure water uptake values measured in this study for different XLPEGDA samples are in excellent agreement with the values previously reported by Ju et al. [16]. 

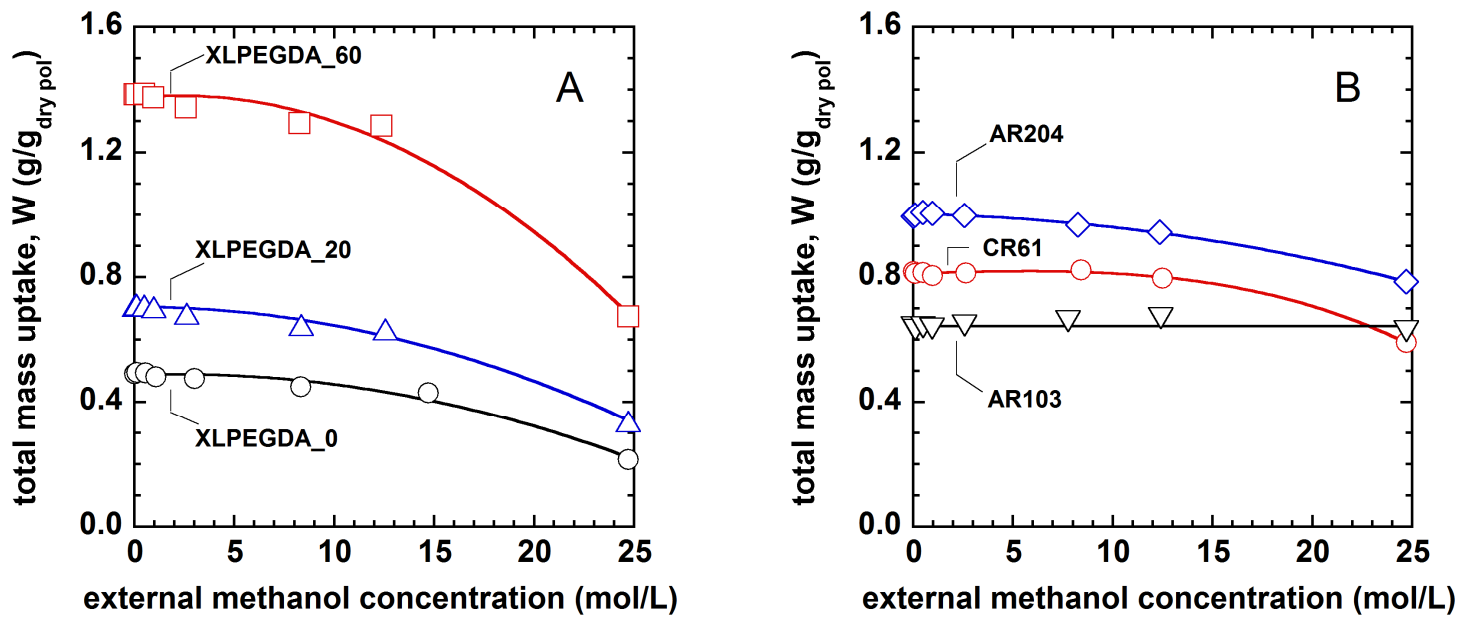

Figure 1. Water-methanol mixture uptake at $25^{\circ} \mathrm{C}$ as a function of external methanol concentration. A) uncharged materials: XLPEGDA_0 (black circles), XLPEGDA_20 (blue triangles) and XLPEGDA_60 (red squares); B) charged materials: AR103 (black triangles), CR61 (red circles) and AR204 (blue diamonds). The continuous lines are guides for the eye.

Qualitatively similar behavior was observed for the cross-linked, charged samples, CR61, AR103 and AR204 (Fig. 1-B). The styrenic membranes, CR61 and AR103, display lower sorption capacity than the acrylic membrane, AR204. This trend presumably reflects effects of differences in cross-linking density and affinity for water or methanol among the three samples. Because both chemical composition and cross-link density are different in each of these samples, we cannot separate these effects on the observed water and methanol uptake properties. Future studies, aimed at producing systematic series of materials with controlled morphology, may shed more fundamental light on this point. The different levels of hydrophilicity displayed by these charged polymers also reflect their different IEC values. As reported in Table 2, CR61 and AR103 have the same nominal value of IEC, which is lower than that of AR204. The greater ion 
exchange capacity of AR204 is consistent with its higher sorption capacity relative to that of CR61 and AR103.

The charged polymers exhibit a smaller difference between water and methanol uptake than the XLPEGDA-based polymers. For example, the total sorption level decreases less than $2 \%$ over the whole range of external methanol concentrations for AR103. For CR61 and AR204, the observed decrease is about $39 \%$ and $27 \%$, respectively. This observation may be associated with the presence of the porous support used with the charged polymers. The porous backing which supports these IEMs may constrain them from swelling as much as they might otherwise do if they were not polymerized into the support, and it may prevent them from freely deswelling at greater external methanol concentrations.

4.2. Methanol and water uptake. From the desorption experiments described earlier, methanol sorption isotherms in charged and uncharged hydrogels were determined. In Fig. 2-A and 2-B, the methanol volume fraction in the charged and uncharged polymers considered in this study is reported as a function of methanol activity in the external solution. Thermodynamic data for water/methanol solutions at $25^{\circ} \mathrm{C}$ have been reported by Kurihara et al. [35] and were used in this study to calculate the methanol activity in the external solution.

The sorption isotherms are nearly linear at low methanol activity, while, especially for the uncharged samples (cf., Fig. 2-A), an upward curvature is observed at higher methanol activity (i.e., at greater external methanol concentration). The methanol content in the XLPEGDA-based polymers increases with increasing amounts of pre-polymerization water (i.e., with decreasing cross-link density). 

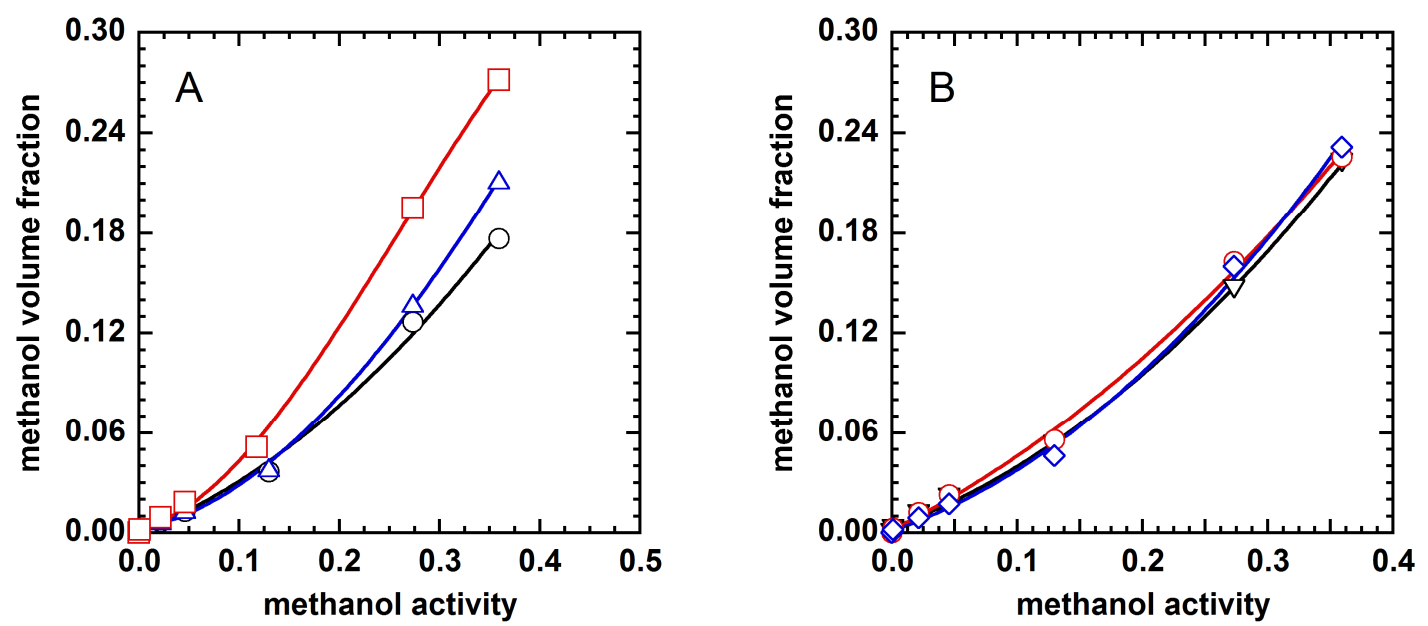

Figure 2. Methanol volume fraction in the swollen polymer as a function of methanol activity in the external solution at $25^{\circ} \mathrm{C}$. A) uncharged materials: XLPEGDA_0 (black circles), XLPEGDA_20 (blue triangles) and XLPEGDA_60 (red squares); B) charged materials: AR103 (black triangles), CR61 (red circles) and AR204 (blue diamonds). The continuous lines are guides for the eye.

The Flory-Rehner model was used to describe the methanol sorption isotherms in XLPEGDA-based polymers. The cross-link density values, $v_{c}$, for XLPEGDA hydrogels prepared with different levels of pre-polymerization water were taken from literature sources [33] and are recorded in Table 1 . As expected, $v_{c}$ decreases with increasing pre-polymerization water content. To minimize the number of adjustable parameters, the methanol-polymer and water-polymer binary interaction parameters were calculated from pure liquid sorption data using the Flory-Rehner equation for a binary (i.e., polymer and one penetrant) system. Conversely, the water-methanol interaction parameter, $\chi_{m w}$, was fit to the experimental mixture sorption data. To get a better representation of the experimental data, $\chi_{m w}$ was allowed to vary with methanol volume fraction in the membrane using the following empirical expression [36]:

$\chi_{m w}=\alpha \phi_{m}+\beta$ 
In summary, the Flory-Rehner model was used to calculate the equilibrium volume fractions of methanol, water and polymer at fixed values of methanol activity in the external soaking solution, with two fitting parameters, $\alpha$ and $\beta$. The best fit values are reported in Table 3 .

Table 3. Values of fitting parameters $\alpha$ and $\beta$ for XLPEGDA polymers.

\begin{tabular}{|c|c|c|}
\hline & $\alpha$ & $\beta$ \\
\hline PEGDA_0 & -4.42 & 2.52 \\
\hline PEGDA_20 & -4.30 & 2.43 \\
\hline PEGDA_60 & -1.72 & 1.95 \\
\hline
\end{tabular}

From the results in Table 1, the methanol-polymer interaction parameter, $\chi_{m p}$, is greater than the water-polymer interaction parameter, $\chi_{w p}$, in each XLPEGDA sample. Thus, waterpolymer interactions should be thermodynamically more favorable than methanol-polymer interactions, which is consistent with the greater total sorption levels in XLPEGDA-based materials in water than in methanol.

Fig. 3 presents methanol, water and polymer volume fractions estimated by the FloryRehner model in XLPEGDA samples as a function of methanol activity in the external solution. The model outcomes are in reasonably good agreement with the experimental data, especially since $\chi_{m p}$ and $\chi_{w p}$ were taken to be constant, not depending on the water/methanol mixture composition. In contrast, to obtain better agreement between this model and experimental measurements of mixed gas carbon dioxide/ethane sorption in XLPEGDA, Ribeiro et al. [36] allowed both the penetrant-polymer and the penetrant-penetrant interaction parameters to be 
non-linear functions of external composition. This assumption, while improving the overall fit of the model to the data, introduces additional fitting parameters, compromising the predictive capability of the model.

The methanol-polymer Flory-Huggins interaction parameter, $\chi_{m p}$, decreases with increasing amount of water in the pre-polymerization solution (cf., Table 1), indicating that methanol interactions with XLPEGDA become more favorable as cross-link density decreases. As reported in Fig. 1-A, samples with lower cross-link density display greater water uptake, so they also provide a more favorable environment for methanol sorption.

In each of the samples shown in Fig. 3, the water volume fraction in the samples decreases as methanol sorption increases. In XLPEGDA_60, more than 60\% of the total sample volume is occupied by water and methanol, while only about $40 \%$ is occupied by polymer. 

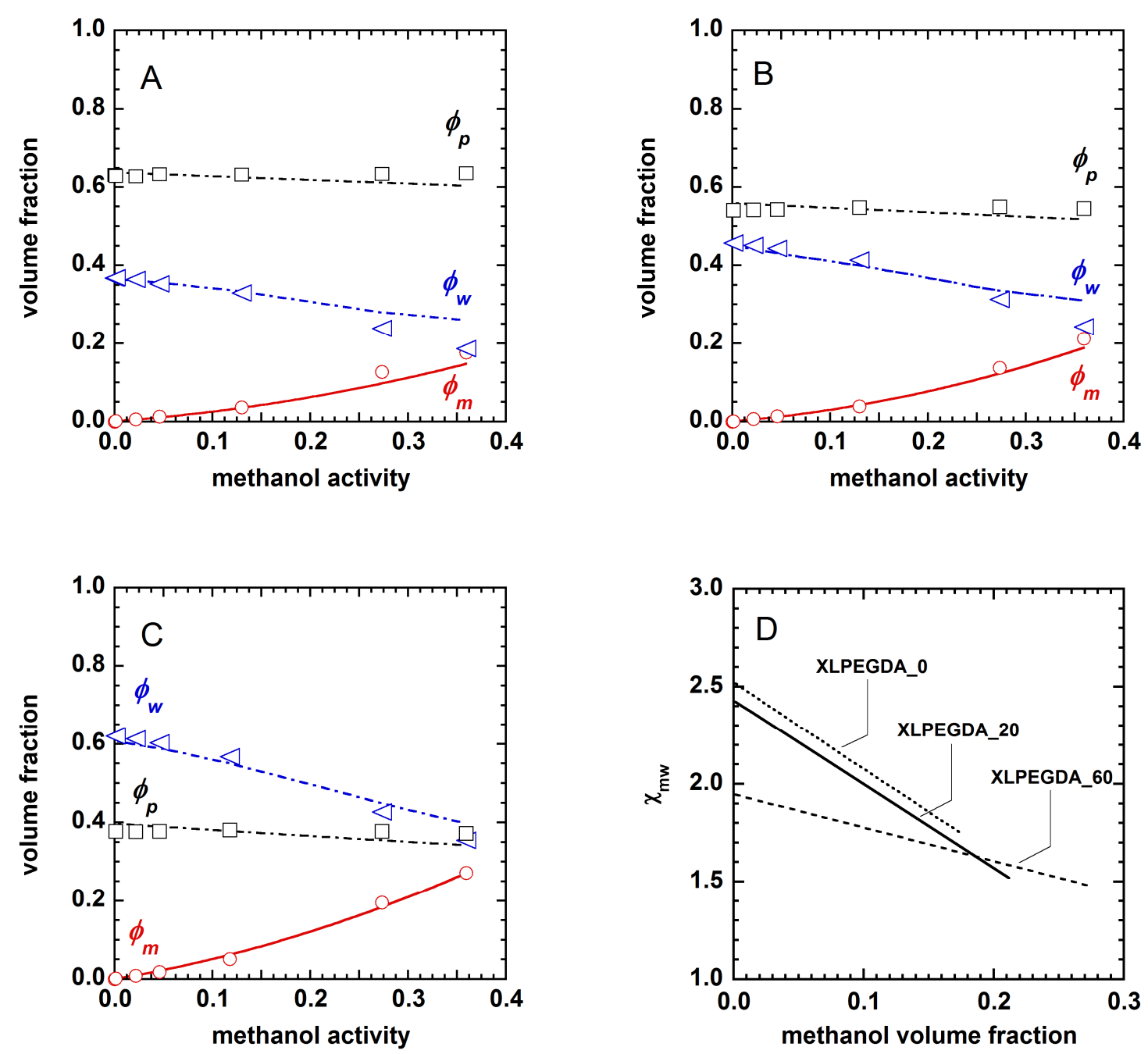

Figure 3. Equilibrium volume fraction of methanol (red circles), water (blue triangles) and polymer (black squares) as a function of methanol activity in the external solution. A): XLPEGDA_0; B): XLPEGDA_20; C): XLPEGDA_60. Continuous and dashed lines: Flory-Rehner model calculations. D): methanol-water Flory-Hugging parameter $\left(\chi_{m w}\right)$ as a function of methanol volume fraction in the polymer.

As shown in Fig. 3-D, the methanol-water interaction parameter, $\chi_{m w}$, obtained by fitting the sorption data to the ternary Flory-Rehner model, is essentially the same in the case of XLPEGDA_0 and XLPEGDA_20, and slightly decreases when considering XLPEGDA_60 at low methanol volume factions. XLPEGDA_60 contains much more water and methanol than 
polymer relative to other XLPEGDA-based materials, which, based on Eq. 13, favors penetrantpenetrant mutual interactions. Moreover, the high water content of XLPEGDA_60 creates a more favorable environment for methanol molecules.

For all XLPEGDA-based polymers considered, the methanol-water interaction parameter is less than its ethanol-water analog, whose average value is 3.5 [32]. On this basis, methanol-water interactions are more favorable than ethanol-water interactions. Since the length of the methanol hydrophobic aliphatic chain is less than that of ethanol, methanol presumably interacts more favorably, from an energetic viewpoint, with water than ethanol does.

Methanol sorption isotherms in charged membranes are reported in Fig. 2-B as a function of methanol activity in the external solution. They also show an upward curvature at high methanol activities, similar to that in XLPEGDA samples (cf., Fig. 2A). Methanol solubility is fairly similar in the three membranes, with AR103 having a slightly lower sorption capacity than the other materials. We did not attempt to use the Flory-Rehner theory to describe sorption isotherms in charged polymers. The cross-link density for these charged membranes is not available, and its experimental estimation is difficult, due to the presence of the porous backing. Moreover, the original model proposed by Flory and Rehner was formulated for neutral crosslinked polymers, not for cross-linked ion exchange polymers [30,31].

An alternative way to present the sorption isotherms is to report the methanol chemical potential in the membrane as a function of penetrant concentration in the membrane [37]. This 
treatment of experimental data is reported and discussed in the Supplementary Information (Fig. S2).

4.3. Methanol permeability and diffusivity. Methanol permeability coefficients in XLPEGDA-based materials are presented in Fig. 4-A as a function of methanol concentration in the donor compartment $\left(C_{m}^{s}\right)$. As already observed for the sorption data, methanol permeability increases with increasing amounts of pre-polymerization water. Samples with greater pre-polymerization water content have lower cross-link density and higher water uptake, which promotes faster methanol transport rates.
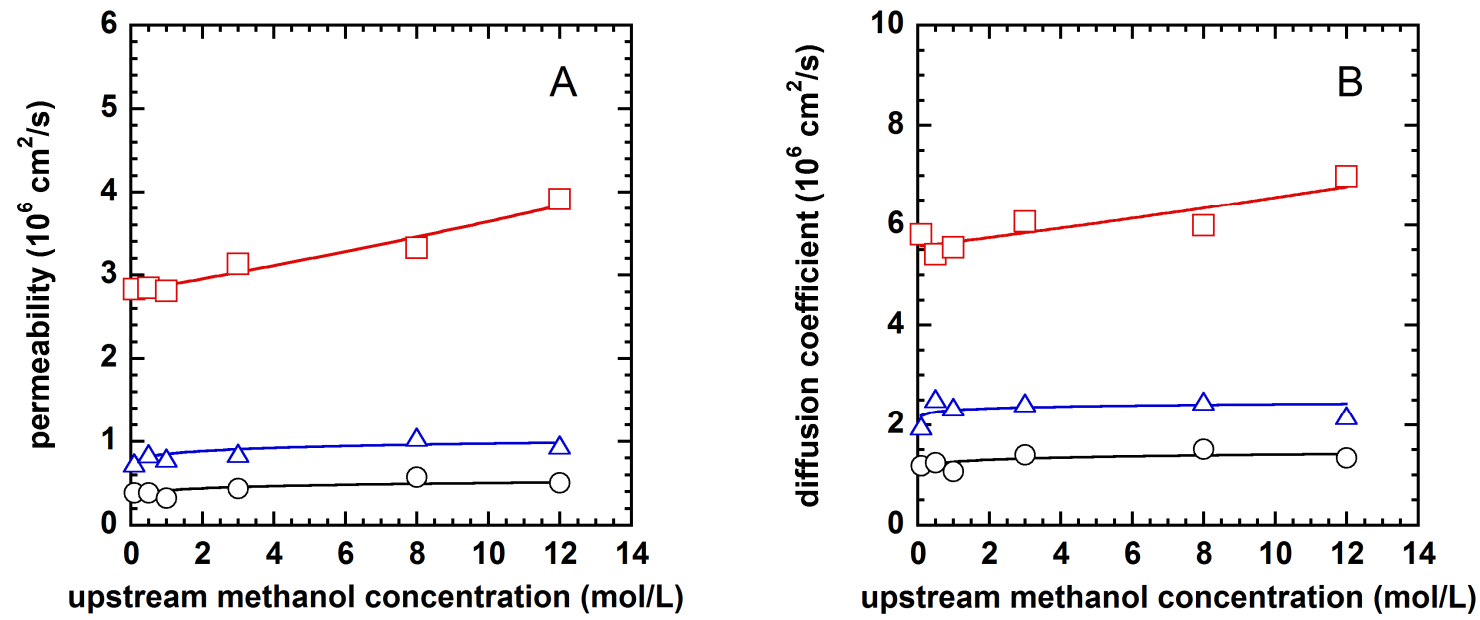

Figure 4. A) Methanol permeability in uncharged hydrogels as a function of methanol concentration in the donor compartment at $25^{\circ} \mathrm{C}$ : XLPEGDA_0 (black circles); XLPEGDA_20 (blue triangles); XLPEGDA_60 (red squares). B) Concentration averaged, effective methanol diffusion coefficient in uncharged hydrogels as a function of methanol concentration in the donor compartment at $25^{\circ} \mathrm{C}$ : XLPEGDA_0 (black circles); XLPEGDA_20 (blue triangles); XLPEGDA_60 (red squares). The diffusion coefficients were corrected for the effects of convection as described in the supplementary information section. The continuous lines are guides for the eye. 
To estimate the diffusion coefficients in highly sorbing systems, it can be useful to separate the influence of convection from diffusion on the total measured flux (see Supplementary Information) [38,39]. This issue has been previously addressed by Paul [40] and Koros et al. [38], in the case of liquid and gas transport in dense membranes. Here, two convective effects on methanol transport have to be considered. The first one is due to water counter-diffusion (i.e., osmotic effects). An additional convective effect comes from the frame of reference. Neglecting the convective terms into the mass balance leads to diffusion coefficients that inherently contain these convection effects [41], so they do not represent the Fickian diffusivity and cannot be directly compared with diffusion coefficients that do not include such effects.

In this study, the methanol diffusion coefficients were corrected for the effects of convection using the approach originally proposed by Paul [40] and reported in the Supplementary Information. The effects of convection become important when the methanol concentration in the membrane is significant (i.e., at high methanol concentrations in the donor compartment (8 and $12 \mathrm{~mol} / \mathrm{L})$ ). In Fig. 4-B, methanol diffusion coefficients in XLPEGDA samples have been estimated as described in the Supplementary Information. Methanol diffusion coefficients increase with increasing water content in the membrane (i.e., XLPEGDA_60 > XLPEGDA_20 > XLPEGDA_0), which is consistent with both the MackieMeares [23] and Yasuda et al. models [28,29]. The uncertainty in the diffusion coefficient data, estimated using the propagation of errors method [42], is about $10 \%$. 
By comparing Fig. 4-A and 4-B, methanol permeability and diffusivity in XLPEGDA_0 and XLPEGDA_20 are nearly independent of external methanol concentration. A moderate increase is observed in XLPEGDA_60, likely due to the significant swelling of this sample in the presence of water and methanol.

Methanol permeability in charged polymers is reported in Fig. 5-A. As with the XLPEGDA-based materials, permeability and uptake are related. For example, AR103 has the lowest sorption capacity and the lowest methanol permeability. Conversely, AR204 exhibits the highest sorption capacity and highest methanol permeability.
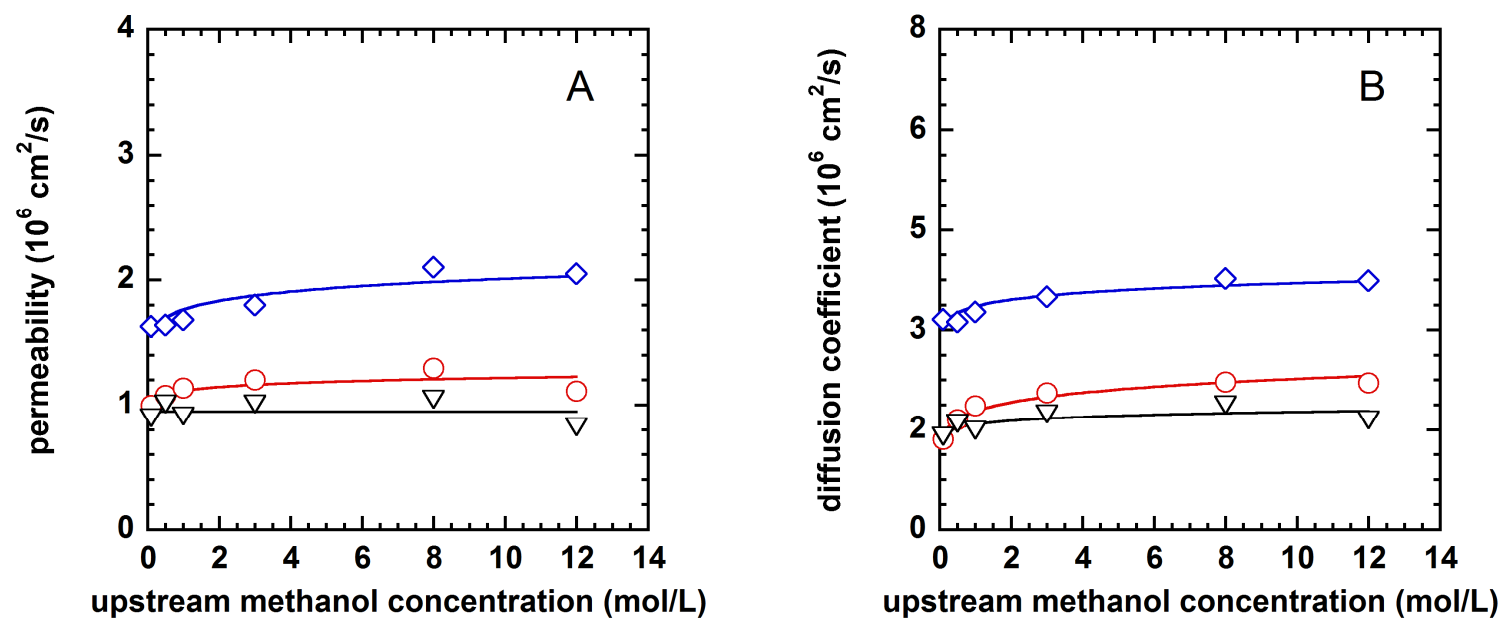

Figure 5. A) Methanol permeability in charged membranes as a function of methanol concentration in the donor compartment at $25^{\circ} \mathrm{C}$ : AR103 (black triangles); CR61 (red circles); AR204 (blue diamonds). B) Methanol diffusivity in charged membranes at $25^{\circ} \mathrm{C}$ : AR103 (black triangles); CR61 (red circles); AR204 (blue diamonds). The continuous lines are guides for the eye.

Methanol diffusion coefficients in the charged polymers (Fig. 5-B) were also corrected for convection, and they exhibit the same qualitative trend observed for permeability. The uncertainty in diffusion coefficients was estimated by the propagation of errors method [42], 
and it is around $10 \%$. Methanol permeability and diffusivity in charged polymers change little with external methanol concentration.

4.4. Modeling methanol diffusivity. The model proposed by Yasuda et al. [28,29] was used to correlate diffusivity data for charged and uncharged polymers. As discussed above, for all the materials considered in this study there is a qualitative correlation between water content and methanol diffusivity, with more swollen membranes having greater methanol diffusivity. A quantitative analysis of this correlation is provided by Eq. 8, which predicts an exponential increase of methanol diffusivity with increasing water content. However, the materials considered in this study sorb a water-methanol mixture, and, in some cases, the methanol sorption is significant. Thus, polymer swelling is caused not only by water, but also by methanol. In this study, we used a modified version of the Yasuda model, in which the water volume fraction in Eq. 8, $\phi_{w}$, was replaced by the sum of water and methanol volume fractions, $\phi_{w}+\phi_{m}$

In Fig. 6A, the experimentally determined methanol diffusion coefficients in the materials considered in this study are presented, on a logarithmic scale, as a function of $\left(\phi_{w}+\phi_{m}\right)^{-1}[28,29]$. A good linear trend is observed, with a correlation coefficient $R^{2}$ of 0.95 , and $A=1.501 \pm 0.1$. Experimental diffusion coefficients for charged and uncharged materials lie on a master-curve. The correlation reported in Fig. 6A includes diffusion data collected at all methanol concentrations considered and for all materials tested in this study.

The Yasuda [28,29] and Mackie-Meares models [23,43] provide similar predictions of methanol diffusion coefficients. As explained earlier, the Mackie-Meares contains no adjustable 
parameters, so it can be used in a completely predictive fashion. The methanol diffusion coefficients can be calculated using Eq. 4, which can be rearranged as follows:

$$
\bar{D}_{m}^{*}=D_{0, m}\left(\frac{\phi_{m}+\phi_{w}}{1-\phi_{m}-\phi_{w}}\right)^{2}
$$

where the volume fraction of polymer, methanol and water can be estimated from the sorption data. The diffusion coefficient, $\bar{D}_{m}^{*}$, appearing in Eq. 14 has been corrected for the convective effects, following the procedure reported in the Supplementary Information, so it does not represents the concentration averaged diffusivity calculated as $P / K$. In Fig. $6 \mathrm{~B}$, the methanol diffusion coefficients calculated using the Yasuda and Mackie-Meares models are reported, along with the experimental data, as a function of $\phi_{w}+\phi_{m}$.
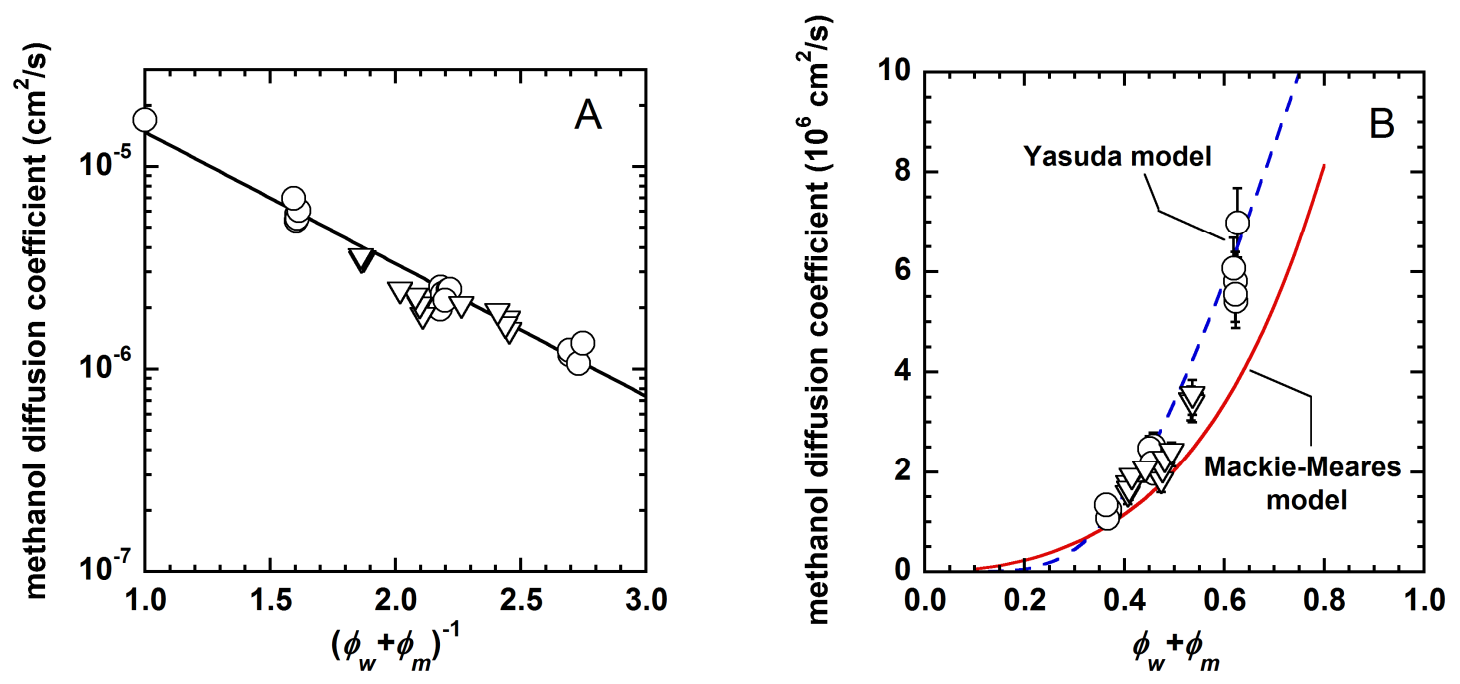

Figure 6. A) Methanol diffusion coefficient as a function of $\left(\phi_{w}+\phi_{m}\right)^{-1}$ in charged (open black triangles) and uncharged membranes (open black circles). Experimental diffusion coefficients were estimated using samples of different materials that had been equilibrated in a mixture of methanol and water. The continuous line represents the best fit of Yasuda's model to the experimental data. When $\left(\phi_{w}+\phi_{m}\right)$ approaches $1, \bar{D}_{m}^{*}=1.5 \times 10^{-5} \mathrm{~cm}^{2} / \mathrm{s}$, which is a good approximation of methanol diffusivity in water. B) Experimental methanol diffusion coefficients in charged (open black triangles) and uncharged materials 
(open black circles). The red continuous line represents the Mackie-Meares model calculations, and the blue dashed line represents Yasuda's model calculations.

Despite its simplicity, the Mackie-Meares model yields a rather good prediction of methanol diffusivity in both the charged and uncharged materials considered in this study. The model's ability to estimate diffusion coefficients in both charged and uncharged polymers suggests that, in the case of ionic hydrogels, the fixed charges do not affect diffusion of a neutral solute, such as methanol, in any special way. These results are consistent with those reported in Fig. 6A, where experimental data for charged and neutral hydrogels lie on the same master-curve. Thus, neutral solute transport rates in charged and uncharged hydrogels are essentially governed by the membrane degree of swelling.

To put these results in perspective, in the Supplementary Information liquid methanol diffusion coefficients in water, glassy thermoplastics and water-swollen hydrogels are compared to each other, and the different mechanism of methanol diffusion in such materials is discussed qualitatively.

4.5. Comparison between methanol and sodium chloride transport. Sodium chloride sorption and permeability coefficients in XLPEGDA_0 at $25^{\circ} \mathrm{C}$ were reported by Geise et al. [19] and Ju et al. [16], respectively, and sodium chloride transport data for CR61 were reported by Kamcev et al. [44]. Solubility and permeability coefficients reported by Kamcev et al. [44] were based on the total volume of composite membrane. That is, they were not corrected for the effect of the 
backing. In this study, these data were re-scaled to the volume of swollen ion exchange polymer, according to the method proposed by Galizia et al. [20].

In Figs. 7, methanol and sodium chloride sorption coefficients at $25^{\circ} \mathrm{C}$ are reported for XLPEGDA_0 and for a CR61 cation exchange membrane. In uncharged polymers, such as XLPEGDA, the sodium chloride sorption coefficient is defined as the ratio of salt concentration in the swollen polymer to that in the external solution [8]. In charged materials, the sorption coefficient is defined as the ratio of the mobile salt concentration in the polymer to the salt concentration in the external solution. For monovalent salt sorption in cation exchange polymers, such as CR61, the mobile salt concentration in the membrane corresponds to that of the co-ion (chloride, in this case) [8].

In uncharged XLPEGDA_0, methanol and sodium chloride solubilities exhibit qualitatively similar behavior when reported as a function of external penetrant concentration (cf., Fig. 7A). These sorption coefficients change little over the concentration range explored. In contrast, methanol and sodium chloride sorption data in the CR61 cation exchange membrane are substantially different (cf., Fig. 7B). The methanol sorption coefficient is nearly independent of external concentration, while a pronounced increase is observed for sodium chloride. These differences arise because sodium chloride sorption in charged materials, like CR61, depends strongly on Donnan exclusion [45]. Concentration driven permeation of salt through charged polymers is largely determined by co-ion mobility, which is chloride in this case [46]. Strong electrostatic interactions between the fixed charges on the polymer backbone and the sodium and chloride ions provide an additional interaction between the penetrant and polymer that is 
not active if either the polymer or penetrant are uncharged. The sharp increase in the chloride sorption coefficient in CR61 as external salt concentration increases is due to Donnan exclusion, which is not present for neutral penetrants, such as methanol, in ion exchange membranes (cf., Fig 7-B). Methanol solubility is greater than that of sodium chloride in all materials considered in this study. Methanol is an organic molecule, so its affinity towards the polymer backbone is greater than that of sodium chloride. Additionally, the solubility of methanol in water is practically infinite, while the solubility of sodium chloride in water at ambient conditions is about $300 \mathrm{mg} / \mathrm{mL}$ [47].
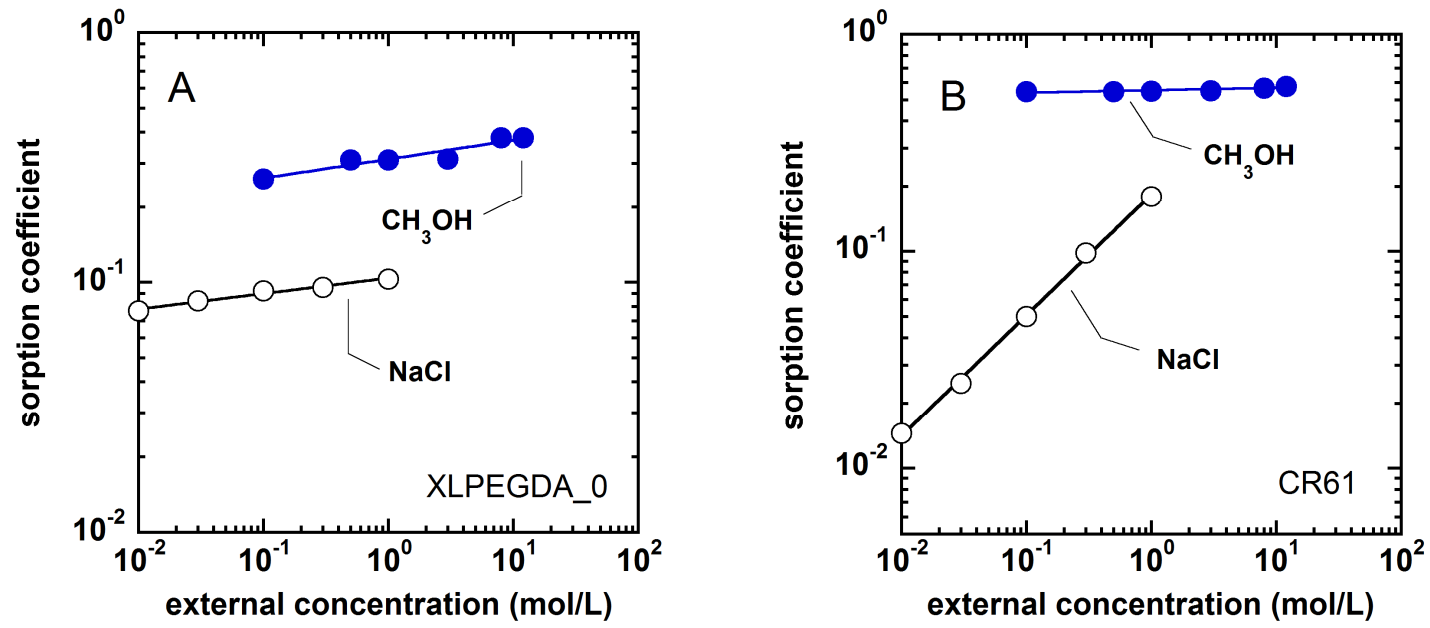

Figure 7. Methanol (blue filled symbols) and sodium chloride (black open symbols) sorption coefficient at $25^{\circ} \mathrm{C}$ in: A) XLPEGDA_0; B) CR61. The lines are a guide for the eye. Sodium chloride sorption data were taken from ref. [44].

As shown in Fig. 8-A, in uncharged XLPEGDA_0, methanol and sodium chloride permeability coefficients exhibit qualitatively similar behavior when reported as a function of upstream methanol or salt concentration. Methanol permeability is about 6 times greater than 
sodium chloride permeability. This difference is mostly due to the sorption contribution, as the methanol sorption coefficient is about 3.5 times greater than that of sodium chloride. The greater methanol permeability is also partially due to the diffusion contribution. The kinetic diameter of methanol is $3.6 \AA$ [48], while the hydrated diameter of $\mathrm{Na}^{+}$and $\mathrm{Cl}^{-}$ions is $7.16 \AA$ and $6.64 \AA$, [49] respectively. Thus, both higher sorption and diffusion contribute to greater permeability by methanol than sodium chloride.

Conversely, in charged membranes [44], sodium chloride permeability increases by an order of magnitude with increasing upstream salt concentration, while methanol permeability is nearly independent of upstream concentration (cf., Fig. 8-B).
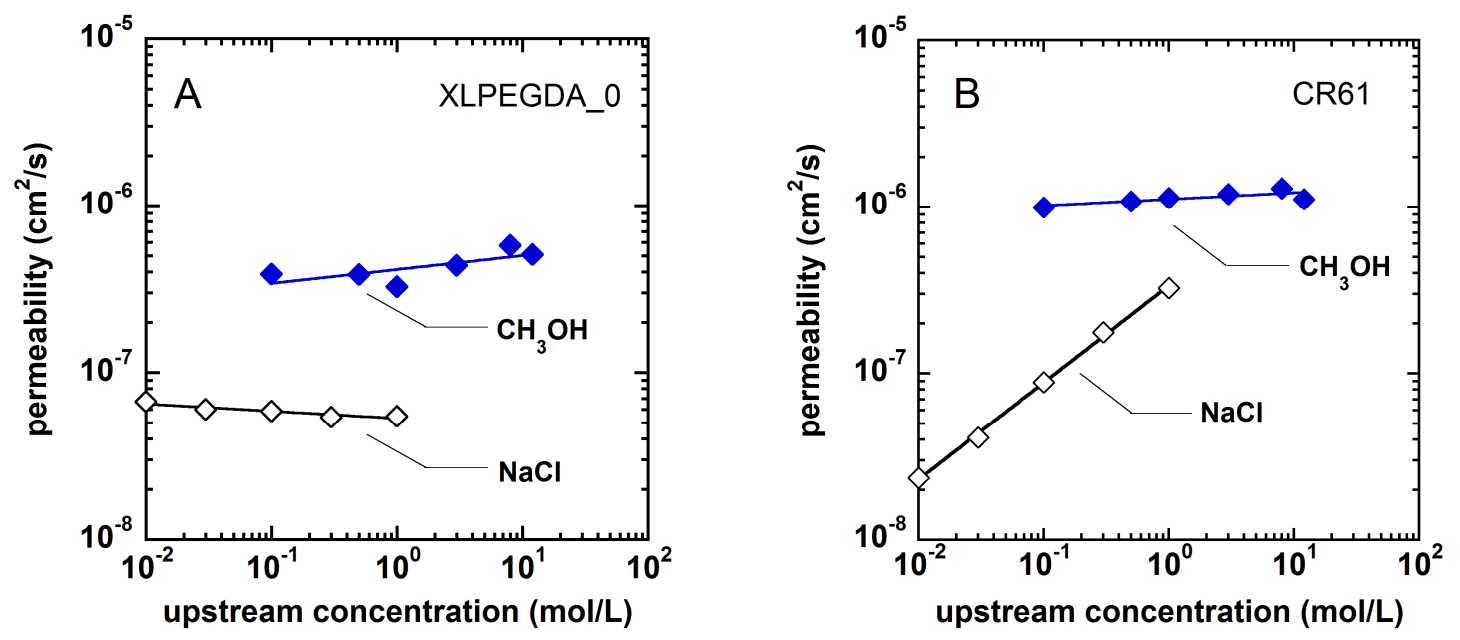

Figure 8. Methanol (blue filled symbols) and sodium chloride (black open symbols) permeability at $25^{\circ} \mathrm{C}$ in: A) XLPEGDA_0; B) CR61. The lines are guides for the eye. Sodium chloride permeability data were taken from ref. [44]. 


\section{Conclusions}

Liquid methanol solubility, diffusivity and permeability in hydrated charged and uncharged polymers have been investigated. Methanol transport is strongly correlated with polymer water content, with materials sorbing more water exhibiting higher methanol transport rates. Both the Mackie-Meares and Yasuda models provide good correlations between methanol diffusion coefficients and the membrane degree of swelling. Sorption of neutral penetrants, such as methanol, in charged and uncharged hydrated polymers occurs by simple thermodynamic partitioning. Conversely, salt transport in charged hydrogels is governed by both thermodynamic partitioning and Donnan exclusion.

The Flory-Rehner model for ternary systems has been used to calculate methanol, water and polymer volume fractions at equilibrium conditions for XLPEGDA-based polymers. The model calculations agree reasonably well with the experimental data.

\section{Acknowledgments}

Partial financial support from the National Science Foundation, Center for Layered Polymeric Systems (Grant 0423914) is gratefully acknowledged. The authors wish to thank General Electric Power and Water for providing the ion exchange membranes used in this work. This study was also partially supported by the International Institute for Carbon Neutral Energy Research (WPI$\left.\mathrm{I}^{2} \mathrm{CNER}\right)$, sponsored by the Japanese Ministry of Education, Culture, Sports, Science and Technology. 


\section{References}

[1] V. Stannett, The transport of gases in synthetic polymeric membranes - an historic perspective, J. Membr. Sci. 19783 97-115

[2] R.W. Baker, Membrane Technology, in Encyclopedia of Polymer Science and Technology, Wiley \& Sons, Hoboken, NJ, 2000

[3] P. Musto, M. Galizia, M. Pannico, G. Scherillo, G. Mensitieri, Time-Resolved Fourier Transform Infrared Spectroscopy, Gravimetry, and Thermodynamic Modeling for a Molecular Level Description of Water Sorption in Poly ( $\varepsilon$-caprolactone), J. Phys. Chem. B 2014118 74147429

[4] M. Galizia, P. Manna, G. Mensitieri, M. Pannico, P. Musto, Diffusion in polymers as investigated by two-dimensional correlation spectroscopy: the $\mathrm{H}_{2} \mathrm{O} / \mathrm{PCL}$ system, J. Molec. Struct. 20141069 290-298

[5] H.B. Hopfenberg, V. Stannett, The diffusion and sorption of gases and vapors in glassy polymers, The Physics of Glassy Polymers, Materials Science Series 1973, 504-547

[6] H. Strathmann, A. Grabowskii, G. Eigenberger, Ion exchange membranes in the chemical process industry, Ind. Eng. Chem. Res. 201352 10364-10379

[7] S.D. Alexandratos, Ion Exchange resin: a retrospective from Industrial and Engineering Chemistry Research, Ind. Eng. Chem. Res. 200948 388-398

[8] G.M. Geise, D.R. Paul, B.D. Freeman, Fundamental water and salt transport properties of polymeric materials, Progr. Polym. Sci. 201439 1-42 
[9] H. Strathmann, Electrodisysis: a mature technology with a multitude of new applications, Desalination 2010264 268-288

[10] L. Li, Y. Wang, Quaternized polyethersulfone cardo anion exchange membranes for direct methanol alkaline fuel cells, J. Membr. Sci. 2005262 1-4

[11] Y. Wei, S. Matar, L. Shen, X. Zhang, Z. Guo, H. Zhu, H. Liu, A novel membrane for DMFC $\mathrm{Na}_{2} \mathrm{Ti}_{3} \mathrm{O}_{7}$ nanotubes/Nafion ${ }^{\circledR}$ composite membrane: performances studies, Int. J. Hydr. En. 2012 37 1857-1864

[12] D.T. Hallinan, Y.A. Elabd, Diffusion and sorption of methanol and water in Nafion using Time-Resolved Fourier Transform Infrared-Attenuated Total Reflectance Spectroscopy, J. Phys. Chem. B 2007111 13221-13230

[13] Y.S. Kim, M.A. Hickner, L. Dong, B.S. Pivovar, J.E. McGrath, Sulfonated poly(arylene ether sulfone) copolymer proton exchange membranes: composition and morphology effects on the methanol permeability, J. Membr. Sci. 2004243 317-326

[14] A. Berger, R.A. Segalman, J. Newman, Material requirements for membrane separators in a water splitting photoelectrochemical cell, Energy Environ. Sci. 20147 1468-1476

[15] M.R. Singh, E.L. Clark, A.T. Bell, Effects of electrolyte, catalyst and membrane composition and operating conditions on the performance of solar driven electrochemical reduction of carbon dioxide, Phys. Chem. Chem. Phys. 201517 18924-18936

[16] H. Ju, A.C. Sagle, B.D. Freeman, J.I. Mardel, A.J. Hill, Characterization of sodium chloride and water transport in crosslinked poly(ethylene oxide) hydrogels, J. Membr. Sci. 2010358 131141 
[17] M. Galizia, Z.P. Smith, G.C. Sarti, B.D. Freeman, D.R. Paul, Predictive calculation of hydrogen and helium solubility in glassy and rubbery polymers, J. Membr. Sci. 2015475 110-121 [18] M. Galizia, M.G. De Angelis, G.C. Sarti, Sorption of hydrocarbons and alcohols in additiontype poly(trimethyl silyl norbornene) and other high free volume glassy polymers. II: NELF model predictions, J. Membr. Sci. 2012 405-406 201-211

[19] G.M. Geise, L.P. Falcon, B.D. Freeman, D.R. Paul, Sodium chloride sorption in sulfonated polymers for membrane applications, J. Membr. Sci. 2012 423-424 195-208

[20] M. Galizia, F.M. Benedetti, D.R. Paul, B.D. Freeman, Monovalent and divalent ion sorption in a cation exchange membrane based on cross-linked poly(p-styrene sulfonate-codivinylbenzene), to be submitted to the Journal of Membrane Science

[21] R.W. Baker, Membrane Transport theory, Membrane Technology and applications, Wiley \& Sons, Hoboken, NJ, 2004, 15-87.

[22] D.R. Paul, Reformulation of the solution-diffusion theory of reverse osmosis, J. Membr. Sci. $2004241371-386$

[23] J.S. Mackie, P. Meares, The diffusion of electrolytes in a cation-exchange resin membrane. I. Theoretical, Proc. Royal Soc. 1955232 498-509

[24] C.R. Wilke, P. Chang, Correlation of diffusion coefficients in dilute solutions, AIChE J. 1955 $1264-270$

[25] D. Green, R. Perry, Chemical Engineer's handbook (7th Ed.), McGraw Hill, New York, 1999 
[26] Y.E. Lee, S.F.Y. Li, Binary diffusion coefficients of the methanol/water system in the temperature range $30-40{ }^{\circ} \mathrm{C}, \mathrm{J}$. Chem. Eng. Data 199136 240-243

[27] J.M. Zielinski, J.L. Duda, Predicting Polymer/Solvent Diffusion Coefficients Using FreeVolume Theory, AIChE J. 199238 405-415

[28] H. Yasuda, C.E. Lamaze, L.D. Ikenberry, Permeability of solutes through hydrated polymer membranes. Part I. Diffusion of sodium chloride, Die Makrom. Chemie 1968118 19-35

[29] H. Yasuda, A. Peterlin, C.K. Colton, K.A. Smith, E.W. Merrill, Permeability of solutes through hydrated polymer membranes. Part III. Theoretical background for the selectivity of dialysis membranes, Die Makrom. Chemie 1969126 177-186

[30] P.J. Flory, J. Rehner, Statistical mechanics of cross-linked polymer networks II. Swelling, J. Chem. Phys. 194311 521-526

[31] P.J. Flory, Statistical mechanics of swelling of network structures, J. Chem. Phys. 195018 $108-111$

[32] E. Favre, Q.T. Nguyen, R. Clement, J. Neel, Application of Flory-Huggins theory to ternary polymer-solvent equilibria: a case study, Eur. Polym. J. 199632 303-309

[33] H. Lin, T. Kai, B.D. Freeman, S. Kalakkunnath, D.S. Kalika, The effects of crosslinking on gas permeability in cross-linked poly(ethylene glycol diacrylate), Macromolecules 200538 83818393

[34] V. Tricoli, Proton and methanol transport in poly(perfluorosulfonate) membranes containing $\mathrm{Cs}^{+}$and $\mathrm{H}^{+}$cations, J. Electrochem. Soc. 1998145 3798-3801 
[35] K. Kurihara, M. Nakamichi, K. Kojima, Isobaric vapor-liquid equilibria for methanol + ethanol + water and the three constituent binary systems, J. Chem. Eng. Data 199338 446-449

[36] C.P. Ribeiro, B.D. Freeman, Carbon dioxide/ethane mixed gas sorption and dilation in a crosslinked poly(ethylene oxide) copolymer, Polymer 201051 1156-1168

[37] M. Galizia, M.G. De Angelis, E. Finkelshtein, Y. Yampolskii, G.C. Sarti, Sorption and transport of hydrocarbons and alcohols in addition-type poly(trimethyl silyl norbornene). I: Experimental data, J. Membr. Sci. 2011 385-386 141-153

[38] H.D. Kamaruddin, W.J. Koros, Some observations about the application of Fick's first law for membrane separation of multicomponent mixtures, J. Membr. Sci. 1997135 147-159

[39] R.B. Bird, W.E. Stewart, E.N. Lightfoot, Transport Phenomena, 2nd Ed., Wiley and Sons, New York, 2005

[40] D.R. Paul, Relation between hydraulic permeability and diffusion in homogeneous swollen membranes, J. Polym. Sci. B: Polym. Phys. 197311 289-296

[41] M.L. White, The permeability of an acrylamide polymer gel, J. Phys. Chem. $1960641563-$ 1565

[42] P.R. Bevington, K.D. Robinson, Data reduction and error analysis for the physical sciences, McGraw Hill, Boston, 2003

[43] D.R. Paul, M. Garcin, W.E. Garmon, Solute diffusion through swollen polymer membranes, J. Appl. Polym. Sci. 197620 609-625 
[44] J. Kamcev, E.S. Yang, N. Yan, D.R. Paul, B.D. Freeman, Effect of ambient carbon dioxide on salt permeability and sorption measurements in ion-exchange membranes, J. Membr. Sci. 2015 $47955-66$

[45] F.G. Donnan, The theory of membrane equilibria, Chem. Rev. 19241 73-90

[46] F. Helfferich, Ion Exchange, Dover Publications, New York, 1995

[47] Sodium chloride data sheet, Sigma Aldrich, www.sigmaaldrich.com

[48] T. Borjigin, F. Sun, J. Zhang, K. Cai, H. Rena, G. Zhu, A microporous metal-organic framework with high stability for GC separation of alcohols from water, Chem. Commun. 2012 $487613-7615$

[49] E.R. Nightingale, Phenomenological theory of ion solvation. Effective radii of hydrated ions, J. Chem. Phys. 195963 1381-1387 